Available online at www.eccomasproceedia.org

Eccomas Proceedia COMPDYN (2021) 1053-1062

ECCOMAS

Proceedia
COMPDYN 2021

$8^{\text {th }}$ ECCOMAS Thematic Conference on Computational Methods in Structural Dynamics and Earthquake Engineering M. Papadrakakis, M. Fragiadakis (eds.) Streamed from Athens, Greece, 28 - 30 June 2021

\title{
CYCLIC TESTING OF 1:40 SCALE CANTILEVER RC ELEMENTS WITH DIGITALLY MANUFACTURED REINFORCEMENT
}

\author{
Lorenzo Del Giudice $^{1}$, Rafal Wrobel ${ }^{2}$, Antonios Katsamakas ${ }^{1}$, Christian Leinenbach ${ }^{2}$, \\ and Michalis Vassiliou ${ }^{2}$
}

${ }^{1}$ ETH Zurich, Swiss Federal Institute of Technology

Institute of Structural Engineering (IBK)

Stefano-Franscini-Platz 5, CH-8093 Zurich

\{delgiudice, katsamakas, vassiliou\}@ibk.baug.ethz.ch

${ }^{2}$ EMPA, Swiss Laboratories for Material Science and Technology

Überlandstrasse 129, CH-8600 Dübendorf

\{rafal.wrobel, christian.leinenbach\}@empa.ch

\begin{abstract}
Time history analysis is considered as the state-of-the-art in modeling of the seismic response of $R C$ structures. Its validation involves predicting the response of an RC structure tested on a shaking table. However, blind prediction contests show that most contestants fail to predict the seismic response of the tested specimens. Given that numerical models are able to accurately capture the behavior of RC members at a component level, we can conclude that a large part of the error sources from the assumptions made to pass from component level to system level, i.e. assumptions related to damping formulation, component interaction, boundary conditions etc. In parallel, the prediction of the response of a structure subjected to a single ground motion has been proven to be too strict of a validation procedure. Oftentimes, a statistical approach involving many specimens and ground motions is necessary. Such an approach is clearly only feasible at a very small scale. At such scales, the reinforcement fabrication and positioning become major issues. We propose to use additive manufacturing technology to digitally fabricate the reinforcement cage necessary for the micro RC element.

This paper presents the results from cyclic tests on 1:40 scale RC cantilever columns. The reinforcing cages were manufactured using a Selective Laser Melting $3 D$ printer that was able to print rebars with submillimeter diameter and yield strength 378MPa. Two different microconcrete mixtures were used based on cement and gypsum. Each sample was reinforced with 18 longitudinal rebars of $0.6 \mathrm{~mm}$ diameter, and $0.35 \mathrm{~mm}$ stirrups with $2.5 \mathrm{~mm}$ of spacing. The cyclic behavior of the columns closely resembles the behavior of full-scale columns indicating that such small-scale specimens can be used of the statistical validation of global level assumptions that numerical models make.
\end{abstract}

Keywords: 3D Printing, Physical Modeling, Earthquake Engineering, Cyclic Test, Micro Reinforced Concrete. 


\section{INTRODUCTION}

Non-linear time history analysis is considered to be the state-of-the-art of structural modeling in earthquake engineering. Its most widespread validation procedure consists of predicting the response of an RC structure tested on a shaking table. However, blind prediction contests show that the contestants fail to predict the response of the tested specimens with reasonable accuracy, even for structures much simpler than the ones constructed in practice, and even when the structural properties and measured excitation are given to the contestants [1-4].

In order to better understand where the error sources from, one can break down the problem into 3 levels: Material, Component, and System Level. In fact, given the behavior at each level, the engineer makes certain assumptions to level-up. For instance, for given material properties the engineer uses a set of assumption, such as beam theory, the interaction of rebars, concrete etc., to reach the component level cyclic behavior. Then, using another set of assumptions (boundary conditions, interaction of components, damping model, numerical integration scheme) we reach system-level dynamic behavior. Blind prediction contests on component level cyclic behavior prove that structural models perform relatively well at this level $[5,6]$. It is the system level behavior that they fail to predict. Therefore, it is reasonable to assume that one main source of error in the structural models at system-level lies in the assumptions made to scale up from component-level cyclic to system-level dynamic behavior. Hence, these need to be improved and, to this end, more system-level tests are needed.

In parallel, it has been claimed that the appropriate way to validate models in earthquake engineering is to perform multiple tests of virgin specimens under the different ground motions that represent the hazard in a given site and to demand that the structural models predict the Cumulative Distribution Function (CDF) of the maxima of the response quantity of interest. This approach has been investigated by Bachmann et al. [7, 8] who have used this statistical validation procedure on the 1963 Housner dynamic response model of an uplifting structure. They performed 600 shaking table tests as well as 600 numerical simulations and they compared both individual test responses and the statistical aggregates of these responses (empirical CDF of the maximum tilt angle). They showed that the 1963 Housner passed the statistical validation test even though it failed the one-to-one validation test. This statistical approach, is not a "good to have", but often a necessity given the often not discussed nonrepeatability of many tests.

Extending the statistical validation to validate the system-level assumptions of RC structures is not trivial since it would require multiple virgin specimens to be tested on a shaking table. This is cost- and time- wise not viable in full scale. To address this problem we suggest performing the statistical validation carrying out multiple tests with small-scale (1:40) specimens in a geotechnical centrifuge. The validation concerns only the system-level assumptions for given and experimentally determined component level behavior. We have also suggested the same method for masonry structures [9].

Reduced scale models of piles and walls are often used in geotechnical engineering to study soil structure interaction, although using different materials. Knappett et al. 2011 [10] and Loli et al. 2014 [11] developed a micro reinforced concrete to study a rocking isolated RC bridge pier at 1:40 scale. Although their results are encouraging, the manufacturing of the $\mathrm{RC}$ elements showed three limitations: the construction time, the accuracy in positioning the reinforcement, and the size limitation of the steel rebars and stirrups. These limitations can be overcome by using a metal 3D printer to manufacture the reinforcement [12]. The use of a metal 3D printer allows manufacturing of the entire reinforcement cage (longitudinal and horizontal reinforcement) with the desired layout. Moreover, these machines can print rebars as small as $200 \mu \mathrm{m}$, which in scale 1:40 correspond to $8 \mathrm{~mm}$. 
This paper aims at characterizing the flexural behavior of 1:40 scale RC columns by means of full-reversed quasi-static cyclic tests. Two cantilever specimens were constructed using two different types of micro-concrete. One sample was manufactured with cement-based micro-concrete (named HSC), and another with a gypsum based micro-concrete (HSG). The tests were designed according to FEMA-461 [13] and a 3D digital image correlation system was used to analyze the strain field during the tests.

\section{MATERIALS AND METHODS}

The flexural behavior of micro $\mathrm{RC}$ components is investigated performing quasi-static cyclic tests on two small-scale (1:40) micro RC columns. The specimens consist of 2 cantilever elements of $15 \times 15 \mathrm{~mm}$ cross section and $52.5 \mathrm{~mm}$ span, with a $65 \times 65 \times 20 \mathrm{~mm}$ base that functions as a footing (Figure 1). Both samples have the same reinforcement, i.e. 18 rebars of $0.6 \mathrm{~mm}$ diameter with $0.35 \mathrm{~mm}$ stirrups with $2.5 \mathrm{~mm}$ spacing, and a concrete cover of $1.64 \mathrm{~mm}$. They differ from each other in terms of the type of micro-concrete used. The HSC Sample ("C" stands for cement) was manufactured using cement based micro-concrete, whereas sample HSG ("G" stands for gypsum) was manufactured with gypsum based micro-concrete. The experiments were performed with a setup that was installed in a Universal Testing Machine (UTM). The measurement system consisted of LVDTs and a 3D-DIC (Digital Image Correlation) system. The latter allowed for the analysis of the displacement and the strain field during the tests.

\subsection{Experimental Setup}

A fully reversed cyclic loading was applied at the top of the samples. The elements were placed horizontally and fixed on a steel support designed to fit in the UTM and to align the samples to the center of the machine (Figure 2).

The base of specimens was fixed to the lateral support with 4 M6 bolts. A $2 \mathrm{~mm}$ steel plate was placed between the bolts and the face of the base to avoid local crushing of the concrete due to compressive force applied by the screws. The load is applied at the centerline of the element by two loading pins, which are connected to the double-hinged fork (Figure 2). The latter transfers the vertical load applied by the UTM. The measuring devices consist of 2 LVTD and a 3D-DIC system. The first LVDT measures the vertical displacement at the application point of the load, namely the loading pins shown in Figure 2, the second LVDT measures the vertical displacement of the base of the element (to measure any possible sliding of the base). The DIC was used to measure the displacements and the strain field at the surface. By measuring the strain distribution, we are able to identify any cracks and microcracks formed during the tests as well as to locate the plastic hinges that formed at the base on the element.

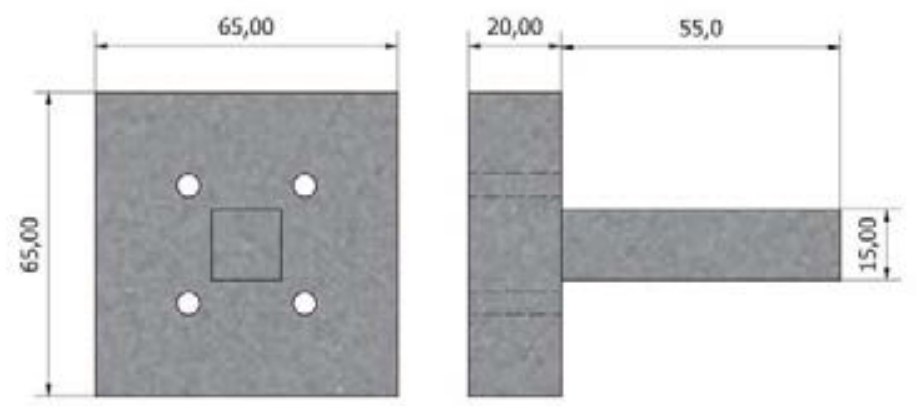

Figure 1. Specimen geometry and dimensions. 


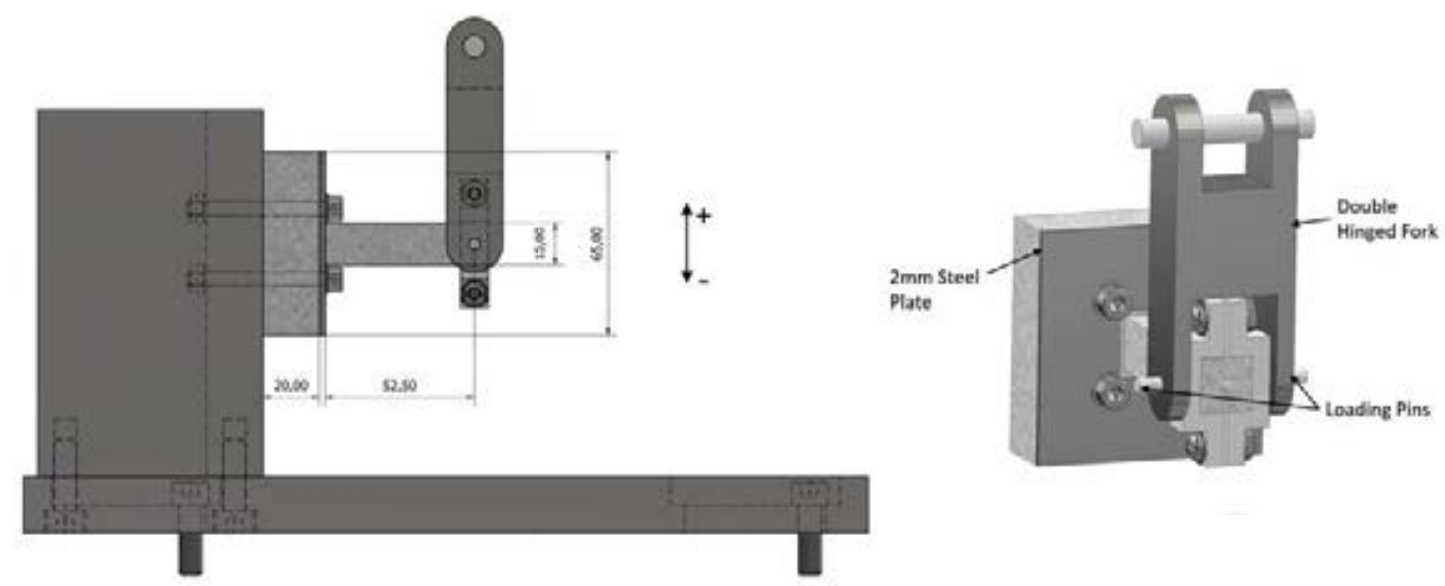

Figure 2. Experimental setup and detail of the loading apparatus.

\subsection{Loading Protocol}

The load is applied by displacement control, applying a displacement $\Delta$ at the top of the elements. Nonetheless, the kinematic parameter that is used to define the loading protocol is the drift ratio $\varphi$, which is defined as the ratio between the top displacement and the element length $l_{v}=52.5 \mathrm{~mm}$ (in this case it also represents the shear length).

The test program is defined based on FEMA-461, in which the amplitude increase is defined as $\varphi_{\mathrm{n}+1}=1.4 * \varphi_{\mathrm{n}}$. However, to capture the behavior of the element in the elastic range, the first amplitude was set equal to $0.5 \times \varphi_{y}$ and the second one was set to the yield drift, $\varphi_{y}$ (Figure 3). The yield drift was calculated for specimen HSC and HSG using a fiber model in Opensees [14]. The micro-concrete was modelled with a Concrete02 constitutive model whereas the 3D printed steel was modeled using Steel02 model. Both material models were calibrated on uniaxial test results that we performed.

The velocity of application of the load was selected considering two principles. First, the displacement needs to be as slow as possible to neglect any inertia effect and to minimize any strain rate effect. Second, the loading rate needs to be reasonably fast to avoid creep and to make the test feasible time-wise. On these premises we started by defining a strain-rate range $\dot{\varepsilon}=10^{-5}-10^{-4} \sec ^{-1}$. This strain refers to the outermost fiber of the cross section of the column that lies closer to the foundation. The lower end of the strain-rate range corresponds to a displacement rate of the top of the column equal to $0.075 \mathrm{~mm} / \mathrm{min}$, which was used for the first three amplitudes of the loading protocol. For the subsequent four amplitudes, the displacement rate was set to $0.413 \mathrm{~mm} / \mathrm{min}$, which corresponds to a strain rate of $5.6 \times 10^{-5} \mathrm{sec}^{-1}$ Finally, the last cycles were applied with a displacement rate of $0.75 \mathrm{~mm} / \mathrm{min}$, corresponding to the upper limit of the aforementioned strain rate range.

\subsection{D Printed Reinforcement}

The use of a 3D printer allows manufacturing of the entire reinforcement cage, namely the longitudinal and shear reinforcement, with the designed layout. The rebars size is not constrained by the manufacturing method. Depending on the $3 \mathrm{D}$ printer, we can print rebars as small as $0.20 \mathrm{~mm}(200 \mu \mathrm{m})$, which in the prototype scale (1:40) represent $\Phi 8$ rebars.

In our study, we used a ConceptLaser M2 Laser Powder Bed Fusion (LPBF) printer. It is able to manufacture various types of metal. For our study, we used gas-atomized stainless steel $316 \mathrm{~L}$ powder with a grain size15-45 $\mu \mathrm{m}$. 


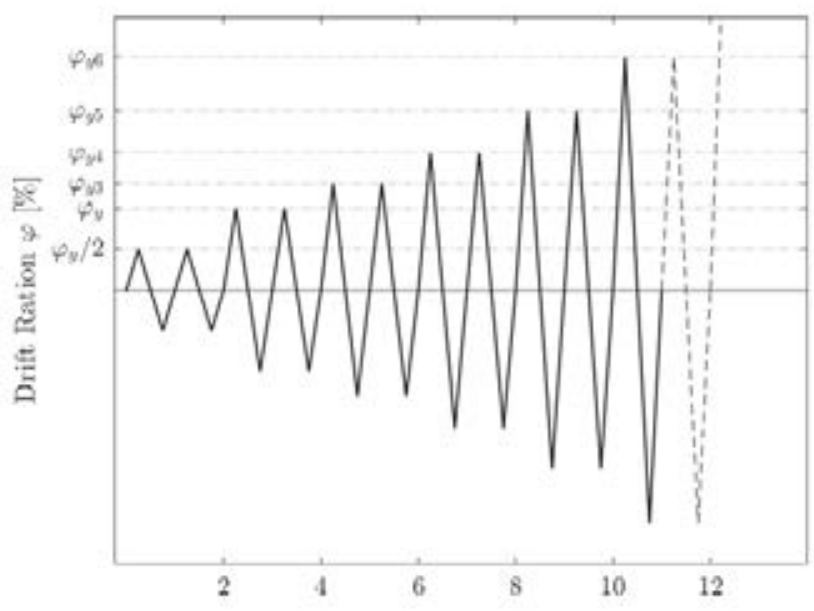

Figure 3. Loading protocol in terms of drift ratio $\varphi$.

We tested specimens with a longitudinal reinforcement ratio $\rho_{l}=3.7 \%$ and a shear reinforcement ratio $\rho_{w}=0.9 \%$. The reinforcement layout consists of 18 bars of $0.6 \mathrm{~mm}$, and $0.35 \mathrm{~mm}$ diameter stirrups with a $2.5 \mathrm{~mm}$ of spacing. In addition, the longitudinal bars were linked with shear ties as shown in Figure 4.

All rebars were printed with ribs on the surface to increase bonding with concrete. In addition, the $3 \mathrm{D}$ printed steel has a roughness $\mathrm{Ra}$ value of $15-20 \mu \mathrm{m}$, hence it has an inherent roughness that is beneficial for the steel-concrete bonding.

The mechanical properties of the $3 \mathrm{D}$ printed steel were characterized with a series on uniaxial tension tests performed on $0.6 \mathrm{~mm}$ diameter bars identical to the ones used as reinforcement in the micro RC samples. Due to the small cross-section, we used a sensor arm extensometer, instead of strain gauges, with a gauge length on $30 \mathrm{~mm}$ to measure the strains during the tests. The resulting average yield strength $f_{y}$ was $378 \mathrm{MPa}$, the average maximum strength $f_{s, \max }$ was $430 \mathrm{MPa}$, and the average Young's Modulus $E_{\mathrm{s}}$ was equal to $180 \mathrm{GPa}$. These values are in line with those reported by Casati et al. 2018 [15] for 3D printed Stainless steel $316 \mathrm{~L}$.
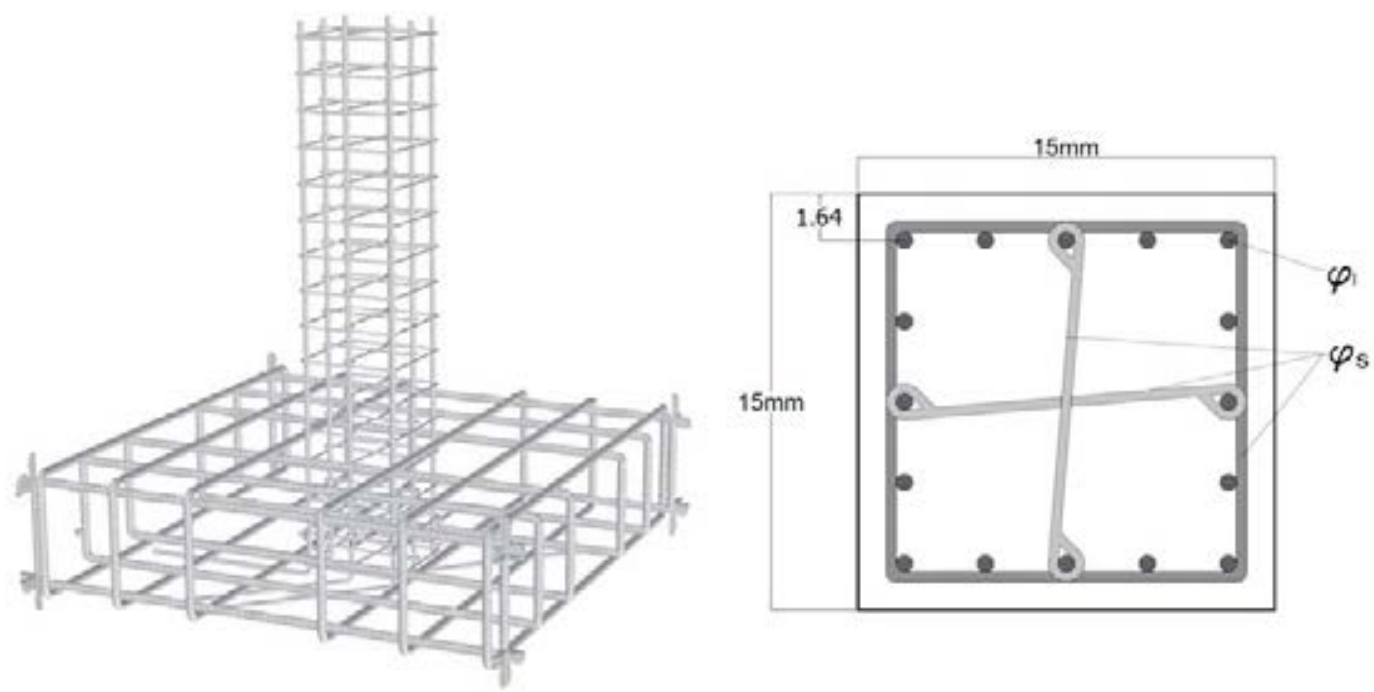

Figure 4. Left: 3D model of the 3D printed reinforcement. Right: Cross section and reinforcement layout. 


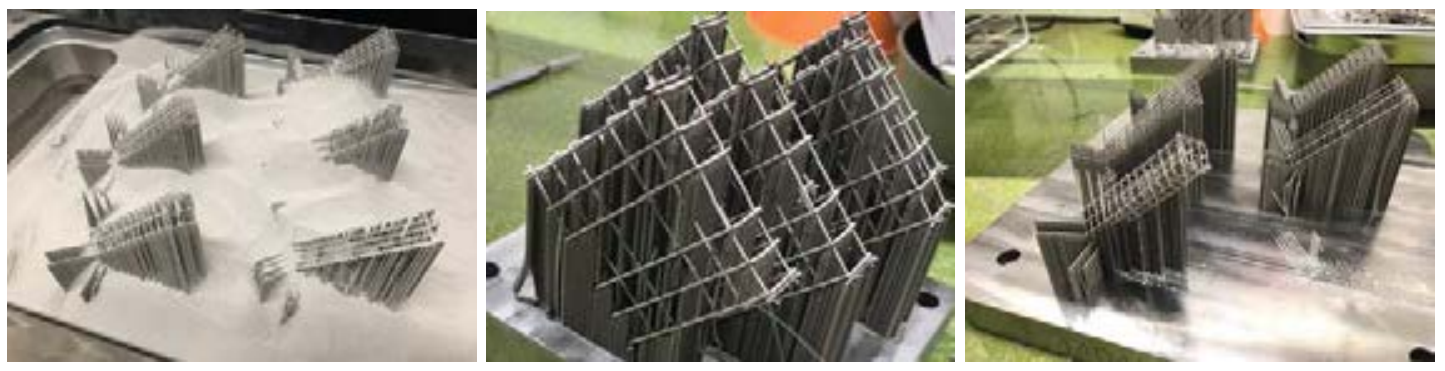

Figure 5. Left: 3D printer Jobbox. Center: Reinforcement cage after the printing process. Right: Footing reinforcement after the printing process.

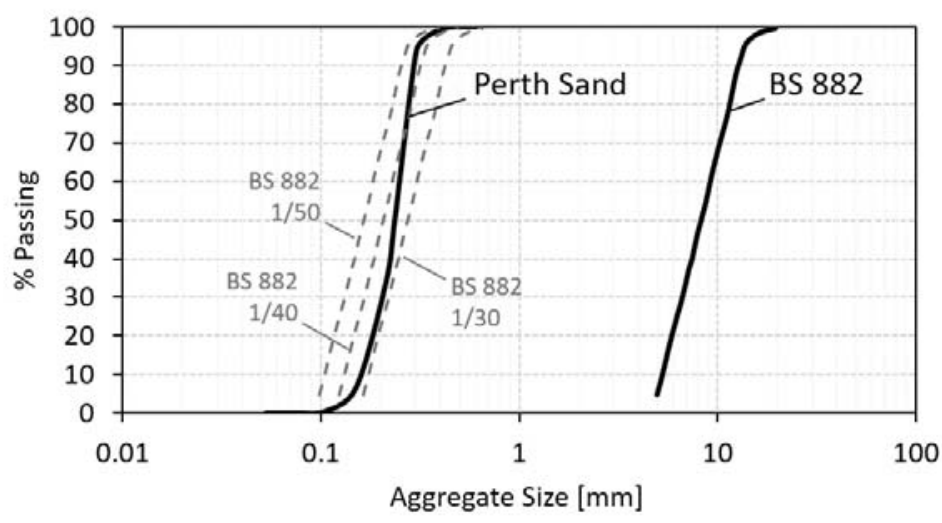

Figure 6. Passing distribution of the sand used for the micro-concrete.

\subsection{Micro Concrete}

Due to the small scale of the specimens, it is necessary to scale the inert component of the concrete so that the ratio inert size / rebars / cross section is about the same as in the real scale. We used Perth silica sand with a d50 $=0.23 \mathrm{~mm}$. Its grain distribution compares very well to the typical aggregate size when scaled 40 times (see Figure 6). Moreover, a different binding agent was studied, alongside the more common cement, i.e. gypsum. The use of gypsum to manufacture micro concrete models is not new, in fact, both Knappett et al. 2011 [10], and Harris et al. 1999 [16] suggest using gypsum to accelerate the curing time and consequently speeding up the specimen manufacturing. Gypsum takes around 48 hours to reach the full strength compared to the usual 28 days for concrete, hence the manufacturing of several specimens for the statistical validation would become considerably faster.

The concrete based micro-concrete used in sample HSC comprises a mixture of cement Normo52.5R, Perth sand, and water. The other mixture, i.e. gypsum based micro-concrete used in HSG, was made of alpha-formed gypsum based plaster (Saint-Gobain Hartform 1) instead on cement. The ratio binding component / sand / water was $1 / 1 / 0.5$ and $1 / 1 / 0.6$ for cement and gypsum based mix respectively. The mix designs were chosen to minimize the water percentage while preserving a good workability and flowability of mixture.

The mechanical properties of both types of micro-concrete were investigated through a series of uniaxial compression tests on cylindrical specimens of $20 \mathrm{~mm}$ diameter and $50 \mathrm{~mm}$ height, and several 4 point bending tests performed on prisms specimens with cross section 15 by $15 \mathrm{~mm}$ and length of $80 \mathrm{~mm}$. Table 1 presents the mean values of the compressive strength $f_{c}$, the strain at the maximum load $\varepsilon_{1}$, the Young's modulus $E_{c}$, and the modulus of rupture $f_{c t}$. 


\begin{tabular}{l|c|c|c|c} 
Micro & $f_{c}$ & $\varepsilon_{1}$ & $f_{c t}$ & $\begin{array}{c}E_{c} \\
\text { Concrete }\end{array}$ \\
{$[M P a]$} & {$[\%]$} & {$[M P a]$} & {$[\mathrm{MPa}]$} \\
\hline Cement & 34.5 & 1.2 & 3.40 & 2089.0 \\
Gypsum & 18.5 & 0.9 & 7.20 & 1109.9 \\
\hline
\end{tabular}

Table 1. Mechanical properties of micro-concrete.

\section{RESULTS}

\subsection{Cyclic Tests}

The lateral force-displacement loops (P- $\Delta$ ) for specimens HSC and HSG are shown in Figures 7 and 8 , respectively. The failure load is conventionally defined as a strength degradation up to $85 \%$ of the peak load.

Specimen HSC exhibits a maximum shear load $F_{\max }=172.80 \mathrm{~N}$ at a drift ratio $\varphi_{\text {Fmax }}=1.47 \%$ and an ultimate strength $F_{0.85}=146.80 \mathrm{~N}$ at a drift ratio $\varphi_{0.85}=3.0 \%$. Yielding occurred at a shear load $F_{y}=115.60 \mathrm{~N}$ at a yield drift ratio $\varphi_{\mathrm{y}}=0.82 \%$. Visual inspection of the sample did not show diffuse cracking on any of the surfaces. Nonetheless, the horizontal strain distribution at peak load, measured with 3D-DIC system, shows tensile strains along the span of the elements HSC (Figure 7, right) that suggest that micro cracks formed during the tests. Regardless of the micro cracks, a localized crack at the base of the element followed by the fracture of the outer longitudinal rebars are to be considered as the causes of failure; no other failure mechanisms could be observed, i.e. concrete spalling.

Specimen HSG reached a maximum load $F_{\max }=184.20 \mathrm{~N}$ at a drift $\varphi_{\mathrm{Fmax}}=4.76 \%$ and ultimate strength $F_{0.85}=156.57 \mathrm{~N}$ at a drift ratio $\varphi_{0.85}=7.65 \%$. The load at yielding $F_{y}$ was equal to $157.40 \mathrm{~N}$ at a yield drift ratio $\varphi_{\mathrm{y}}$ of $1.58 \%$. The hysteresis loop in Figure 8 shows a clear pinching behavior, unlike the loop of the HSG sample. The strain distribution at peak load did not show concentrated tensile strains anywhere along the specimen unlike what was observed in specimen HSC. The tensile strains concentrated at the base of the element. This confirms what was observed visually, namely that the failure was caused by a localized crack at the base. Similarly to HSC, in specimen HSG no concrete spalling was observed. Comparing the two results, the sample with cement based micro-concrete resulted in a stiffer and less ductile behavior than the sample with gypsum based micro-concrete. Moreover, cracks are more diffused in the HSC micro RC specimen.

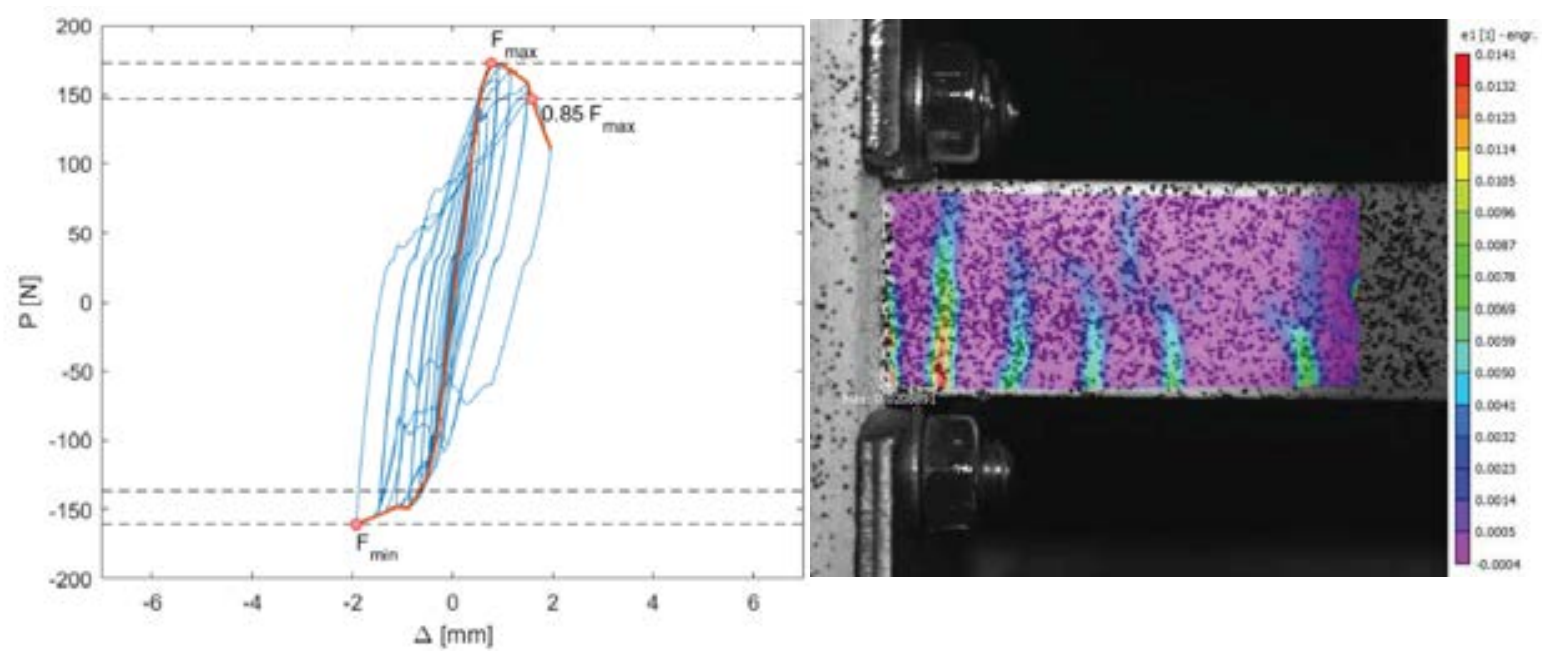

Figure 7. Left: HSC Hysteresis loop and backbone curve. Right: Horizontal strain field at maximum load. 


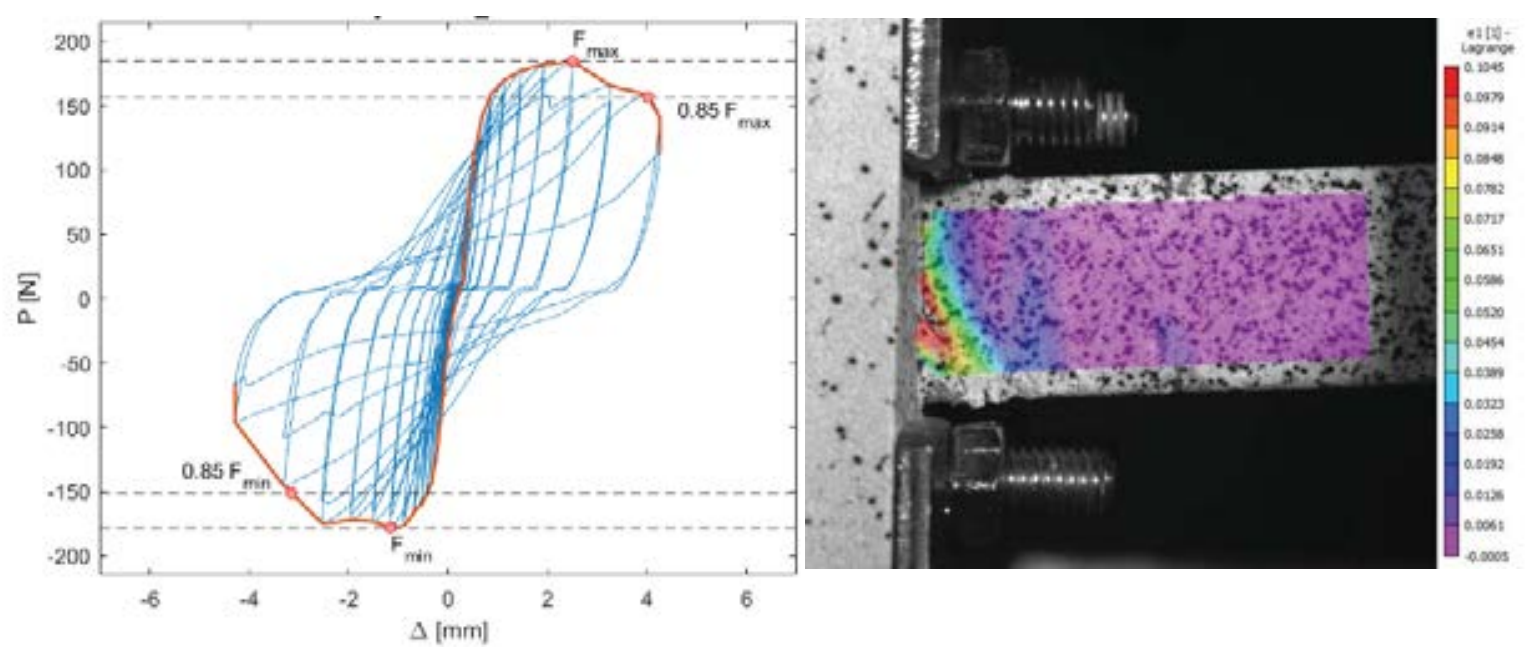

Figure 8. Left: HSG Hysteresis loop and backbone curve. Right: Horizontal strain field at maximum load.

\begin{tabular}{c|c} 
Parameter & Value \\
\hline$f_{y}$ & $378[M P a]$ \\
$E_{s}$ & $180[G P a]$ \\
$b_{s}$ & 0.004 \\
$\varepsilon$ lim & 0.09 \\
\hline
\end{tabular}

Table 2. Steel02 parameters used in the Opensees model.

\begin{tabular}{c|c|c|c|c|c} 
Specimen & $f_{c}[\mathrm{MPa}]$ & $\varepsilon_{1}$ & $f_{c c}[M P a]$ & $\varepsilon c c$ & $f_{c c u}[M P a]$ \\
\hline HSC & 34.5 & 0.012 & 43.16 & 0.027 & 42.64 \\
HSG & 185 & 0.009 & 26.81 & 0.029 & 21.24 \\
\hline
\end{tabular}

Table 3. Concrete 02 parameters used in the Opensees model. The properties $f_{c c}, \varepsilon c c$, and $f_{c c u}$ refer to the confined concrete of the section core.

\subsection{Numerical Model}

Numerical models of the cyclic tests were implemented in Opensees, and the results were compared against the experimental curves. The specimens were modelled as cantilever beams with a fixed support. This assumption was confirmed by the DIC measurements that showed no rotation at the connection of the elements with their footings. The reinforcement was modeled using the OpenseesnSteel02 model, and the strain at failure Elim was modeled using the MinMax model. The parameters used are summarized in Table 2.

The Opensees model used for concrete is Concrete 02 with different parameter values for the core and the cover material. The latter was calibrated based on the material level tests on the small-scale specimens, whereas the core concrete properties were calculated using Mander's model [16]. The model parameters are summarized in Table 3.

Figure 9 (left) shows the comparison between the experimental and numerical results of the HSC specimen. The numerical simulation is able to capture the maximum load measured during the test, as well as the strength degradation of the post elastic behavior. The stiffness degradation in the numerical curve is also remarkably similar to the experimental curve. On the other end, the numerical simulation of specimen HSG (see Figure 9, right) fails to capture key values, such as the peak load, the strength degradation, and most of all the stiffness degradation. In fact, the simulation does not show any pinching behavior unlike the experimental curve of HSG. 

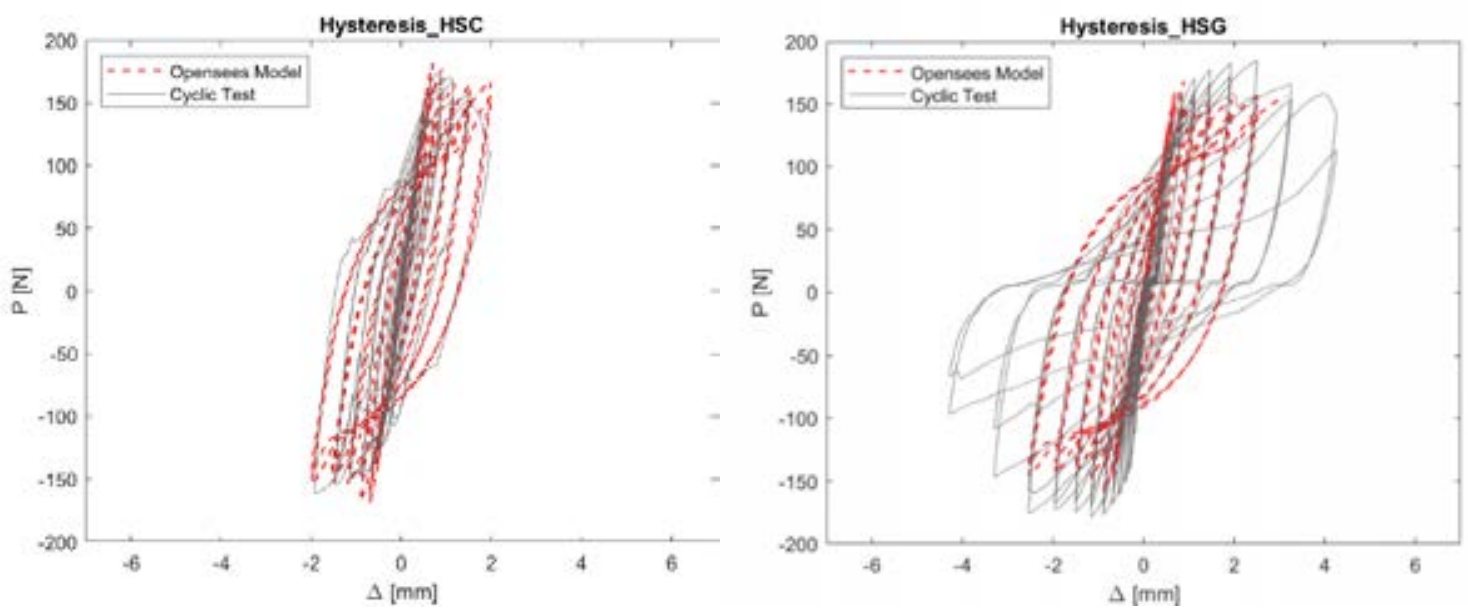

Figure 9. Comparison between experimental curves and Opensees models.

\section{CONCLUSION}

Additively manufactured reinforcement allows the production on multiple small-scale reinforced concrete specimens. The tested elements show a flexural behavior, which is similar to full size prototype RC structural members. Cement based micro-concrete has a compressive and tensile strength similar to concrete, unlike gypsum based micro-concrete that has a tensile strength substantially higher than concrete. Moreover, the numerical simulations in the case of specimen HSC (cement base micro-concrete) are able to accurately capture the experimental behavior. The numerical model of specimen HSG does not match the behavior observed experimentally, as it could not capture neither the peak strength nor the pinching behavior of the tested specimen. Further component level tests are required to fully characterize the flexural behavior of micro RC specimens. Nonetheless, it seems feasible to produce small-scale models of a full structure to perform dynamic tests in a geotechnical centrifuge. The shake table tests could provide a dataset to statistically validate the assumptions that are usually made to scale up from component-level cyclic to system-level dynamic behavior.

\section{REFERENCES}

[1] M. Schoettler, J. Restrepo, G. Guerrini, D. E. Duck, A full-scale, single-column bridge bent tested by shake-table excitation, 2012.

[2] V. Terzic, M. J. Schoettler, J. I. Restrepo, S. A. Mahin, Concrete column blind prediction contest 2010: outcomes and observations, PEER Report, 1, 1-145, 2015.

[3] M. Panagiotou and J. I. Restrepo, Displacement-based method of analysis for regular reinforced-concrete wall buildings: Application to a full-scale 7-story building slice tested at UC-San Diego, Journal of Structural Engineering, 137, no. 6, 677-690, 2011.

[4] M. Panagiotou, J. I. Restrepo, and J. P. Conte, Shake-table test of a full-scale 7-story building slice. Phase I: Rectangular wall, Journal of Structural Engineering, 137, no. 6, 691-704, 2011.

[5] M. Trüb, Numerical modeling of high performance fiber reinforced cementitious composites, IBK Bericht, vol. 333, 2011.

[6] X. Lin and X. Lu, Numerical Models to Predict the Collapse Behavior of RC Columns and Frames, The Open Civil Engineering Journal, 11, no. 1, 2017. 
[7] J. A. Bachmann, M. Strand, M. F. Vassiliou, M. Broccardo, and B. Stojadinović, Is rocking motion predictable?, Earthquake Engineering \& Structural Dynamics, 47, no. 2, 535-552, 2018, doi:1 0.1002/eqe.2978.

[8] J. Bachmann, M. Strand, M. F. Vassiliou, M. Broccardo, B. Stojadinovic, Modelling of rocking structures: Are our models good enough? 2nd International Conference on Natural Hazards \& Infrastructure (ICONHIC 2019), Chania, Crete, Greece, June 23-26, 2019.

[9] Del Giudice, L.,Vassiliou, M. F. Mechanical properties of 3D printed material with binder jet technology and potential applications of additive manufacturing in seismic testing of structures. Additive Manufacturing, 36, 101714, 2020.

[10] J. A. Knappett, C. Reid, S. Kinmond, K. O'Reilly, Small-scale modeling of reinforced concrete structural elements for use in a geotechnical centrifuge, Journal of Structural Engineering, 137, no. 11, 1263-1271, 2011.

[11] M. Loli, J. A. Knappett, M. J. Brown, I. Anastasopoulos, G. Gazetas, Centrifuge modeling of rocking-isolated inelastic RC bridge piers, Earthquake engineering \& structural dynamics, 43, no. 15, 2341-2359, 2014.

[12] Del Giudice, L., Wrobel, R., Leinenbach, C., and Vassiliou, M. F. Static testing of additively manufactured microreinforced concrete specimens for statistical structural model validation at a small scale. 8th International Conference on Advances in Experimental Structural Engineering (8AESE), Christchurch, New Zealand, February 3-5. 2020.

[13] FEMA, A. 461/Interim Testing Protocols for Determining the Seismic Performance Characteristics of Structural and Nonstructural Components. Applied Technology Council, Redwood City, CA, 113. 2007

[14] Mazzoni, S., McKenna, F., Scott, M. H., Fenves, G. L. OpenSees command language manual. Pacific Earthquake Engineering Research (PEER) Center, 264, 2006.

[15] R. Casati, J. Lemke, M. Vedani, Microstructure and fracture behavior of 316L austenitic stainless steel produced by selective laser melting, Journal of Materials Science \& Technology, 32, no. 8, 738-744, 2016.

[16] Harris, H. G., Sabnis, G. Structural modeling and experimental techniques. CRC press, 1999.

[17] J. B. Mander, M. J. N. Priestley, R. Park, Theoretical stress-strain model for confined concrete, Journal of structural engineering, 114, no. 8, 1804-1826, 1988. 